\title{
Robust NVH Engineering using Experimental Methods - Source Characterization techniques for Component Transfer Path Analysis and Virtual Acoustic Prototyping
}

\author{
Kevin Wienen and Michael Sturm Robert Bosch Automotive Steering LLC \\ Andy Moorhouse and Joshua Meggitt University of Salford
}

\begin{abstract}
A major challenge in automotive NVH engineering is to approach complex structure-borne sound and vibration problems with sufficient accuracy but reasonable experimental effort. Typical issues encountered are poor correlation between objective component performance criteria tested for during bench validation and corresponding subjective targets evaluated during system validation in the actual vehicle. Additional challenges arise from the need to impose assumptions on sophisticated physical vibration problems to reduce the complexity to a level feasible for conventional experimental test methods. This paper addresses all mentioned issues by elaborating on a system NVH engineering approach employing Virtual Acoustic Prototyping (VAP) (related to what is now often called component Transfer Path Analysis) to synthesize time domain sound and vibration responses of vibrating machinery operated in a virtual vehicle environment. One crucial step of VAP is to characterize the strength of vibrating machinery by independent quantities at the significant coupling degrees of freedom (DoF). This study puts special focus on the measurement of free velocity, suitable for machinery operated when resiliently mounted as per ISO 9611, and the in-situ measurement of blocked forces, applicable for sources connected to any type of receiving structure during operation, as per ISO/DIS 20270. In order to reduce complexity of the underlying measurements this paper investigates the possibility of using collocated sensor arrays and methods to validate assumptions imposed to abstract away from rotational coupling DoF. An electric power steering (EPS) system inducing vibrations into a sub-frametype structure is considered as a representative automotive sourcereceiver installation to investigate the feasibility of free velocity and in-situ blocked force approach with respect to independent source characterization for component Transfer Path Analysis (TPA) and VAP. The obtained Virtual Acoustic Prototype is expanded using an algorithm to synthesize realistic time domain data, enabling $\mathrm{NVH}$ engineers to conduct reliable objective and subjective design evaluations.
\end{abstract}

\section{Introduction}

Rapidly increasing product complexity together with quickly evolving customer expectations on functionality, performance and quality attributes drive manufacturers to shorten product development cycles whilst the number of product variants increases. To minimize costs and time to market a reoccurring trend of fewer physical prototypes towards virtual system validation can be observed. As far as automotive NVH engineering is concerned, Virtual Acoustic Prototyping (VAP) is considered the most suitable process to predict, auralize, diagnose and assess vibro-acoustic performance in a virtual vehicle environment, in lieu of testing and validating $\mathrm{NVH}$ performance in physical prototypes. VAP involves the construction of a virtual assembly from the properties of its constituent active and passive vibro-acoustic components [1]. Preferably, these properties are described by an independent characteristic of the active vibration source at its coupling interface and corresponding structural dynamic properties of the passive assembly [2]. In a few cases, these characteristics can be found by modelling, but most practical applications require measurement approaches to determine active and passive characteristics within a sufficient frequency range. Experimental methods, however, only provide an approximation of the true system, thus, introducing errors contributing to the overall uncertainty in the VAP predictions

Recent advancements in the fields of component Transfer Path Analysis (TPA) [3] and Dynamic Substructuring [4] are steadily driving change with respect to addressing challenges associated with VAP techniques. Regarding agreed standards on experimental methods to perform independent source characterization, the measurement of free velocity, suitable for machinery operated when resiliently mounted, as per ISO 9611 [5], has been in use for more than a decade. The involved measurements are relatively simple to realize and restricted to the coupling interface DoF only. In response to demand from mechanical industries to provide a standardized procedure applicable to machinery that cannot be operated under (approximated) free boundary conditions, the in-situ blocked force method, proposed by Moorhouse et al. [6], is now adopted in ISO/DIS 20270 [7]. The standard specifies a procedure to inversely determine blocked forces with the source being connected to an arbitrary receiver. Due to the invariant property of the blocked force, source data measured in-situ is transferable between different assemblies. This allows comparing sources operated under similar conditions, but in different assemblies (e.g. bench and vehicle), or employing blocked forces from one installation (e.g. bench) to predict vibration in or sound radiated from a different source-receiver combination (e.g. vehicle). The latter application constitutes the presented VAP framework. However, involving an inverse procedure implicates sensitivity of the in-situ blocked force method to inconsistencies in the measured input data. The location (fully or partially coinciding with the coupling interface DoF) and the number (degree of over-determination) of the indicator DoF at which such input data is measured, significantly influence the solution of the generally ill-posed inverse problem. In recent work, guidelines for instrumentation to reduce uncertainty are given in [8], and [9] provides a framework for the evaluation of uncertainties in blocked forces. However, application of this framework is as yet nonstandard. On the other hand, the uncertainties in free velocity can be evaluated directly [5]. The most practical approach to validate the reliability of inversely determined blocked forces, at current state of the art, was found to be an on-board validation approach [7] carried

Page 1 of 12 
out in parallel to the in-situ measurement as adopted in ISO/DIS 20270.

A problem experimentalist applying either of the two standards have to face, is to balance experimental effort against completeness of the measurement in terms of describing the physical vibro-acoustic problem. Both methods assume point contact at the coupling interface of the source and facilitate considering 6 coordinate DoF (3 translations and 3 rotations) per contact point. In theory, a complete interface description must account for all of these [10]. Although experimental methods are available to sufficiently approximate translational and rotational DoF [11], even at inaccessible points of structures [12, 13], applying such methods requires skilled experimentalists; not to mention the considerable experimental effort and required instrumentation. For many industrial applications and due to certain practical limitations or purposes it is favorable to consider reducing the number of coupling interface DoF [5]. To verify if the coupling interface is sufficiently described after such reduction, ISO 9611 suggests cumbersome sound transmission tests for the free velocity measurement requiring the source including isolators being connected to a number of typical receiver structures. A recent study by Meggitt et al. [10] proposes the use of the so-called Interface Completeness Criteria (ICC) to address problems related to interface incompleteness in coupled structures. Derived from the insitu blocked force theory [6, 12], the ICC approach relies on measurements of the dynamic properties of the assembly which could easily be obtained during a preliminary validation test with artificial source excitation as suggested by ISO/DIS 20270. Thus, a quantitative assessment of the degree to which the coupling interface has been described during the in-situ blocked force characterization comes at very little costs in terms of additional experimental effort. On this account, the ICC method seems to be a promising approach towards validating assumptions imposed on the coupling interface DoF when attempting to deliberately trade accuracy against simplicity of the in-situ blocked force measurement (ISO/DIS 20270), e.g. when deciding on neglecting rotational DoF in industrial applications.

Recent research has provided evidence that employing diagnostic procedures and predictive approaches related to in-situ blocked force component TPA [14-17] and VAP [2, 18] can improve robustness in the NVH development process of automotive structure-borne sound and vibration sources. However, the high complexity in the involved experimental source characterization step potentially prohibits adopting such methods to assess NVH performance for multiple parts every day, as often required for testing and validation purposes in the automotive sector. This paper seeks to remedy this problem by investigating potentials to simplify the source characterization step embedded in the frameworks of component TPA and VAP. Using the example of a Rack-and-pinion Electric Power Steering (REPS) system inducing vibrations in 3 translational directions via 3 rigid mounts into a subframe-type receiver, the feasibility of free velocity (ISO 9611) and in-situ blocked force measurement (ISO/DIS 20270) for independent source characterization is investigated. Whilst considering the free velocity approach as benchmark in terms of least measurement effort, different sensor arrangements are explored in the in-situ blocked force characterization to account for indicator DoF coinciding partially or fully with the coupling DoF of the REPS. The different source characterization results then are compared in terms of experimental effort and accuracy. In order to identify uncertainty in the measured blocked forces associated with data inconsistency and interface incompleteness, on-board validation and the Interface Completeness Criteria are applied, respectively. In a subsequent step, all obtained source data are combined with assembly FRFs from a different subframe installation in order to adopt the VAP framework to predict and auralize structure-borne sound when the REPS system is operated in a virtual vehicle environment. In order to investigate uncertainty associated with transferability of the source data, VAP prediction results are compared with actual measurements in frequency and time domain. Finally, source path contribution analysis of the steering induced structure-borne sound is conducted in the virtual prototype, so as to benchmark the suitability of free velocity and in-situ blocked force source characterization for a typical automotive VAP application.

\section{Theory}

Notwithstanding that VAP has been in use for some years in the automotive industry [19], no accepted standard has yet been adopted. Challenges in adopting a standard VAP approach relate to considerations regarding

1. agreed experimental methods to independently characterize the 'strength' of a vibration source at its coupling interface DoF,

2. uncertainties in such source description and the way this data is transferred to predict sound and vibration in other assemblies; particularly when imposing assumptions on coupling DoF to deliberately trade accuracy against simplicity of the method (e.g. discretization of continuous coupling interface into a finite number of points or neglect of rotational DoF), and

3. appropriate data processing techniques to create virtual acoustic prototypes capable of synthesizing realistic time domain predictions suitable for objective and subjective design studies in virtual vehicle assemblies and sub-assemblies.

Theoretical background and methods to address these challenges, as relevant for this paper, are provided in the corresponding sections.

\section{Independent source characterization for TPA und VAP}

Quantities that independently characterize sources of structure-borne sound and vibration are the free velocity and the blocked force. The blocked force is the dynamic force an operating source would exert through its contact interface when constrained by a perfectly rigid foundation [7]. Hypothetically, it can be measured directly, but bulky test rigs are required to approximate true blocked terminations over the frequency range of interest [17]. The free velocity, on the contrary, describes the activity of a vibration source in terms of the motion at its contact interface whilst operated uncoupled and freely suspended. To provide a complete characterization of the source the measurement of free velocity must be accompanied by the source's passive properties, i.e. its free mobility [20]. For a vibration source (A), described by a finite number of contact DoF at interface (c), the independent vector of blocked forces, $\overline{\mathbf{f}}_{A, c}$, and the corresponding vector of the free velocities, $\tilde{\mathbf{v}}_{A, c}$, are related by

$$
\tilde{\mathbf{v}}_{A, c}=\mathbf{Y}_{A, c c} \overline{\mathbf{f}}_{A, c}
$$

or, when solved for the blocked force vector

$$
\overline{\mathbf{f}}_{A, c}=\mathbf{Y}_{A, c c}^{-1} \tilde{\mathbf{v}}_{A, c}
$$

where $\mathbf{Y}_{A, c c}$ is the free mobility matrix of the unconstrained coupling interface DoF. An experimental approach to determine translational and angular free velocity at the source's coupling interface DoF is specified in ISO 9611 [5]. To approximate free suspension, the

Page 2 of 12 
standard requires vibration sources to be operated when mounted on resilient mounts (isolators) separate from any rigid support. In practice, this requirement prohibits characterization of sources running under load or rather to account for internal excitation mechanisms inside active components that may vary with external loading [2]. Moreover, as with the direct measurement of blocked forces, acquiring free velocity as per ISO 9611 only applies for sources that can physically be separated from an installation.

For applications in which it is not intended or not feasible to characterize the source separately from a (rigidly) connected receiver, the so -called (vibro-acoustic) 'transfer path problem' [3] has to be solved. Figure 1 provides an explanation of the problem in the context of in-situ blocked forces, as relevant for this paper.

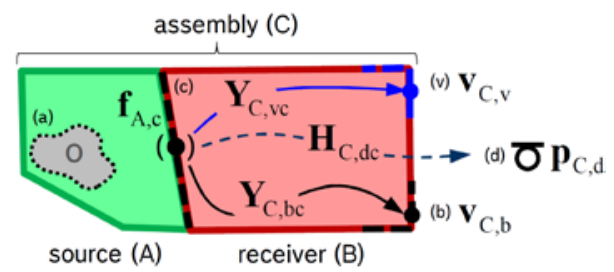

Figure 1. Illustration of the transfer path problem in the context of in-situ blocked forces: A vibration source (A), e.g. steering system, connected to a passive receiver (B), e.g. vehicle, causes vibration $\left(\mathbf{v}_{\mathrm{C}}\right)$ in, or structure-borne sound $\left(\mathbf{p}_{C, d}\right)$ radiated from, the assembly $(\mathrm{C})$ at interfaces $(\mathrm{b}, \mathrm{v})$ and $(\mathrm{d})$, respectively. The internal excitation at (a) is unknown, requiring the source to be characterized at the contact interface (c) by blocked forces $\mathbf{f}_{A, c}$, inferred from $\mathbf{v}_{c, b}$ and the assembly FRF matrix $\mathbf{Y}_{C, b c}$. Additional structural, $\mathbf{Y}_{C, v c}$, and vibro-acoustic FRFs, $\mathbf{H}_{C, d c}$, can be used for prediction and validation purposes.

The in-situ blocked force method [6] is a promising approach to solve the transfer path problem in Figure 1 using a two-stage measurement in which source (A) and receiver (B) remain connected. In the first step, frequency response functions (FRFs) between the coupling interface degrees of freedom (c) and the indicator DoF (b) are determined, yielding the transfer mobility matrix, $\mathbf{Y}_{C, b c}$, of the assembly $(C=A+B)$. If more convenient, this matrix can be measured reciprocally, as $\mathbf{Y}_{C, c b}^{T}$. In a second step, the vibration source is operated and the vector of operational velocity responses, $\mathbf{v}_{C, b}$, is measured at the receiver indicator interface (b). The vector of complex operational blocked forces of source (A), $\overline{\mathbf{f}}_{A, c}$, then is obtained from

$$
\overline{\mathbf{f}}_{A, c}=\mathbf{Y}_{C, b c}^{+} \mathbf{v}_{C, b}
$$

the symbol '+' denoting Moore-Penrose pseudo inverse since $\mathbf{Y}_{C, b c}$ may not be square. If accessible for measurements, in-situ blocked forces can also be determined as per

$$
\overline{\mathbf{f}}_{A, c}=\mathbf{Y}_{C, c c}^{-1} \mathbf{v}_{C, c}
$$

where $\mathbf{Y}_{C, c c}$ is the square and symmetric point mobility matrix of DoF at (c) and $\mathbf{v}_{C, c}$ is the corresponding velocity vector. Partitioned matrices and vectors can be used to combine Eq. (3) and Eq. (4)

$$
\overline{\mathbf{f}}_{A, c}=\left[\frac{\mathbf{Y}_{C, c c}}{\mathbf{Y}_{C, c b}^{T}}\right]^{+}\left\{\frac{\mathbf{v}_{C, c}}{\mathbf{v}_{C, b}}\right\}
$$

On-board Validation (OBV) should be utilized to check the reliability of the inversely identified blocked force vector. As illustrated in Figure 1, OBV predicts additional validation responses at DoF $v_{1}$ from a subset $\left\{v_{1} \subseteq v\right\}$ of the receiver responses $(v)$ within the same source-receiver installation (C)

$$
\hat{\mathbf{v}}_{C, v 1}=\mathbf{Y}_{C, c v 1}^{T} \overline{\mathbf{f}}_{A, c}
$$

The predicted responses, $\hat{\mathbf{v}}_{C, v 1}$, then are compared to actual measurements, $\mathbf{v}_{C, v 1}$, to quantify errors included in the blocked force vector.

This (validated) blocked force then can be used to estimate target vibrations at DoF $v_{2}\left\{v_{2} \subseteq v\right\}, \hat{\mathbf{v}}_{C, v 2}$, and/or to predict sound pressure responses, $\widehat{\mathbf{p}}_{C, d}$, in the same source-receiver installations, as per

$$
\left\{\frac{\hat{\mathbf{v}}_{C, v 2}}{\widehat{\mathbf{p}}_{C, d}}\right\}=\left[\frac{\mathbf{Y}_{C, c v 2}^{T}}{\mathbf{H}_{C, c d}^{T}}\right] \overline{\mathbf{f}}_{A, c}
$$

where $\mathbf{Y}_{C, c v_{2}}$ and $\mathbf{H}^{T}{ }_{C, c d}$ are the vibration and noise transfer functions matrices of assembly (C), measured reciprocally in most practical applications. A practical implementation of inverse blocked force synthesis in Eq. (3-5) in combination with the prediction step in Eq. (7) is known as 'in-situ Transfer Path Analysis' [15] or 'component blocked-force TPA' [3]. Several case studies confirm the suitability of component blocked-force TPA for diagnostics of $\mathrm{NVH}$ problems in complex automotive assemblies [2, 15, 16, 18, 21]. In cases in which component TPA is benchmarked against classic (matrix inverse) TPA, in-situ TPA was found superior in terms of experimental effort at no cost in terms of reliability [2, 15].

Due to the invariant property of the blocked force, independent source data, $\overline{\mathbf{f}}_{A, c}$, can be 'transferred' to a different installations (C') to synthesize vibration, $\widehat{\mathbf{v}}_{C^{\prime}, v}$, in and radiated sound, $\widehat{\mathbf{p}}_{C^{\prime}, d}$, from the new receiver structure (B') when source (A) is virtually operated

$$
\left\{\begin{array}{l}
\hat{\mathbf{v}}_{C^{\prime}, v} \\
\hat{\mathbf{p}}_{C^{\prime}, d}
\end{array}=\left[\frac{\mathbf{\mathbf { Y }}_{C^{\prime}, v c}}{\mathbf{H}_{C^{\prime}, d c}}\right] \overline{\mathbf{f}}_{A, c} .\right.
$$

Here, $\mathbf{Y}_{C^{\prime}, v c}$ and $\mathbf{H}_{C^{\prime}, d c}$ describe the structural and vibro-acoustic frequency response functions (FRFs) of assembly $\left(C^{\prime}=A+B\right.$ ') that may be obtained by in-situ FRF measurement (if the actual assembly (C') is available), experimental dynamic substructuring [4] (if an individual set of FRFs is available for the substructures of source (A) and receiver (B')) or suitable numerical approaches.

The process of synthesizing responses in a virtual assembly (Eq. (8)) from independent characteristics of the active vibration source and dynamic properties of the passive assembly is referred to as Virtual Acoustic Prototyping. The VAP approach adopted in this study relies on experimental data raising questions about uncertainty in the prediction results as discussed in the following.

\section{On the qualitative assessment of uncertainty}

Considering that different sources of errors contribute to the overall uncertainty in the VAP prediction, experimenters would benefit from methods to identify errors inherent in the measured source strength, as well as, errors induced by transferring this data to predict sound and vibration in different assemblies. For most standardized test methods, uncertainty of the former kind can be expressed in some direct way, e.g. by estimated standard deviations for the free velocity measurement uncertainty as per ISO 9611[5]. However, the uncertainties in blocked forces have to be evaluated indirectly [9]. On this account, ISO/DIS 20270 [7] suggests the use of the on-board validation approach (Eq. (5)) with the attempt to identify significant

Page 3 of 12 
errors in the inversely determined blocked forces. Satisfying onboard validation results, however, do not necessarily guarantee transferability of the obtained blocked forces [7] or identification of potential problems associated with an incomplete description of the coupling interface, e.g. due to neglecting rotational DoF. The latter is referred to as 'interface completeness problem' [10].

Considering transferability of source data first, potential prediction problems are likely to be reduced by considering the representativeness of the receiver as per ISO/DIS 20270. In other words, the receiver used for the in-situ blocked force characterization (Eq. (3-5)) shall dynamically be similar to that of the intended installation (e.g. Eq. (8) for VAP), particularly at the sensitive source-receiver interface. It is noted that similar considerations are generally not possible for the transfer of free velocity source data due to the idealized free boundary conditions. Furthermore, before predictions in assembled structures are possible, for compatibility reasons, the measured free velocity data has to be converted into blocked forces using measurements of the source's free driving-point mobilities (Eq. (2)). Thus, additional errors related to inconsistency and incompleteness in the experimental data are expected to further reduce reliability of the VAP prediction. A practical approach to qualitatively assess transferability uncertainty of blocked forces determined in an assembly $(\mathrm{C}=\mathrm{A}+\mathrm{B})$ is to adopt the on-board validation approach from Eq. (6) in order to compare predicted responses with an actual measurement in the target installation $\left(C^{\prime}=A+B\right.$ '). In essence, such transferability validation is already accounted for by Eq. (8), if it is possible to acquire vibration measurements for a subset $v_{t}\left\{v_{t} \subseteq v\right\}$ of the receiver responses $(v)$. Since vibration measurements from the actual target installation are required, this transferability validation is of limited use with respect to VAP. However, for cases in which an actual installation is accessible, the method can potentially help to assess the reliability of the VAP predictions.

Pertaining finally to the challenge of identifying erroneous source data from incomplete descriptions of the coupling interface. An interesting solution to the interface completeness problem is provided by Meggitt et al in [10]. Derived from in-situ blocked force theory [6] and relationships for coupled structures [12], a coherence-style criterion is proposed to assess the completeness of a given interface description. This so-called Interface Completeness Criteria (ICC) is defined as

$$
\operatorname{ICC}_{b a}=\frac{\left|\mathbf{Y}_{b a}^{c}\left(\mathbf{Y}_{b a}^{c_{i}}\right)^{H}\right|^{2}}{\mathbf{Y}_{b a}^{c}\left(\mathbf{Y}_{b a}^{c}\right)^{H} \mathbf{Y}_{b a}^{c_{i}}\left(\mathbf{Y}_{b a}^{c_{i}}\right)^{H}}
$$

where, $\mathbf{Y}_{b a}^{\left(c_{i}\right)}=\mathbf{Y}_{b c_{i}} \mathbf{Y}_{c_{i} c_{i}}^{-1} \mathbf{Y}_{c_{i} a}$.

The coherence-style ICC $\mathrm{C}_{\mathrm{ba}}$ estimates the degree of model uncertainty presented in a given incomplete interface description through the mathematical blocking of a transfer function measured between a set of source-side DoF, $a$, and a single receiver-side DoF, $b$, (see Figure 1 for clarity). As such, depending on the contribution of the coupling DoF a different ICC $_{b a}$ is obtained for each remote receiver DoF considered [10]. In order to excite all significant modes of the coupling interface DoF it is recommended to excite the assembly in at least 6 not coincided source-side DoF to obtain a multi-directional artificial excitation representative for the intended operation and source mechanisms.

Page 4 of 12
In the context of VAP the Interface Completeness Criteria enables to validate assumptions imposed to abstract away from a realistic interface characterization, such as the neglect of rotational or in-plane coupling DoF, in the attempt to reduce the measurement effort of the in-situ blocked force. A further assumption routinely encountered in practice is the abstraction of the continuous contact surface to discrete point-like interface contacts. In both cases this criterion may be used to check whether sufficient coupling DoF have been included in the source characterization setup yielding an indicator for the completeness of the experimental data intrinsic to the source.

\section{VAP and component (blocked force) TPA framework}

Based on the previous discussions, the framework, depicted in Figure 2, is adopted.

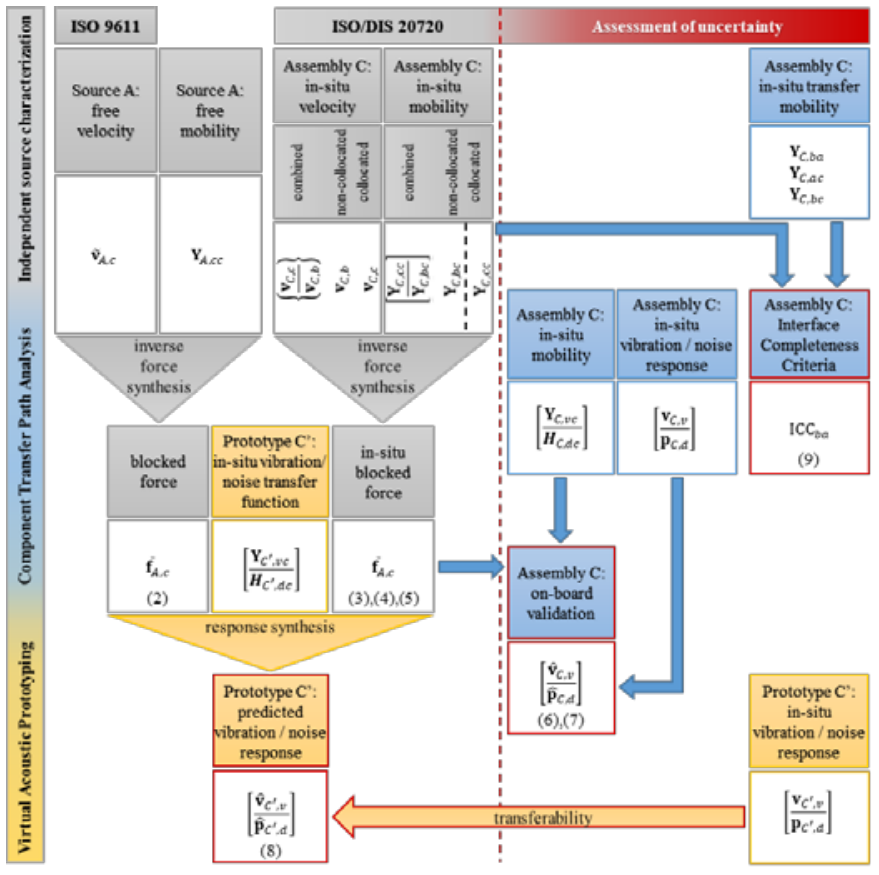

Figure 2. Framework to conduct component TPA and VAP using independent source data from free velocity (ISO 9611) and in-situ blocked force (ISO/DIS 20270) measurements with collocated, non-collocated or combined sensor arrays. Validation approaches facilitate the assessment of uncertainty related to incompleteness, inconsistency and transferability. Numbers in the diagram refer to corresponding equations in the text.

In an initial characterization step, inverse force synthesis is employed to describe the activity of the vibration source at its coupling interface DoF by receiver-independent blocked forces. This characterization is conducted in agreement with

1. ISO 9611 [5]: the measurement of (free) velocity for machinery operated when resiliently mounted, requiring an additional characterization of the source's free driving-point mobilities, and

2. ISO/DIS 20270 [7]: the indirect measurement of (in-situ) blocked forces for sources connected to arbitrary receivers.

In subsequent steps, the obtained blocked forces are combined with corresponding FRFs of the same or a different source-receiver assembly to predict the vibration and/or sound response in the actual or a virtual installation, respectively. Response synthesis of the former type is denoted by component TPA while the latter is denoted by VAP (Figure 2). 
Experimental validation approaches are implemented in the framework to qualitatively assess uncertainty in the VAP predictions related to interface incompleteness, data inconsistency and problems with the transferability of independent source data between assemblies.

Considerations on sensor arrangement: The ill-posed nature of the inverse force synthesis problem requires special care to improve the conditioning. Experimentally, this relates to sensor placement and sensor quantity [8] with additional constraints imposed by the selected measurement approach. For better readability of Figure 2, both factors (placement and quantity), are generalized with respect to the associated sensor arrangement. Hence, the term 'collocated' sensor array is used if response indicator DoF coincide with the source contact DoF, for instance in the inverse problem of Eq. (2) for the free velocity approach. The term 'non-collocated' is used if all sensors are located remotely from the coupling DoF, according to the in-situ blocked force inverse problem of Eq. (3). If a combination of the two sensor arrays is used, i.e. sensors are located at, and remote from the coupling interface DoF, the term 'combined' sensor array is adopted.

\section{Experimental results}

This section investigates possibilities to reduce the effort associated with experimental methods to predict, auralize and diagnose structure-borne sound and/or vibration in coupled structures according to the process adopted in the framework of component (blocked force) TPA and VAP (Figure 2).

In an experimental setup, depicted in Figure 3, a Rack-and-pinion Electric Power Steering (REPS) is considered as active vibration source (A). Its continuous contact interface consists of 3 rigid mounts, approximated by the (point-like) coupling points ' $\mathrm{CP} 1$ ' to 'CP3', each described by 3 translational DoF ( $\mathrm{x}, \mathrm{y}, \mathrm{z})$. To achieve repeatable operational conditions, the REPS is artificially excited on the housing of the electric motor by an electro-magnetic shaker, or alternatively by controlled signals provided directly to the electric motor in order to simulate time-variant steering maneuvers [22].

The REPS can be connected to two different subframe receivers. Receiver structure (B) is built from multiple parts of a vehicle subframe and rigidly connected to ring-fixtures (see Figure 2(a)). Receiver (B') is dynamically more flexible (downstream the contact interface) since suspended freely (see Figure 2(c)). Different sourcereceiver constellations are investigated:

Original assembly $(\mathbf{C}=\mathbf{A}+\mathbf{B})$ : The in-situ blocked force procedure, as per ISO/DIS 20270, is applied in the original assembly (C), consisting of REPS connected to receiver (B). The sensitivity of the in-situ blocked force on sensor placement and degrees of overdetermination is investigated using different sensor arrays. The driving point dynamics at the source contact DoF, for instance, are described by a determined 9x9 (square) mobility matrix (Eq. 4), denoted by the term 'collocated'. The so-called 'non-collocated' array includes only transfer mobilities measured reciprocally between the indicator DoF and the contact DoF arranged in a 2 fold overdetermined 18x9 (rectangular) transfer mobility matrix (Eq. 3). Finally, in-situ blocked forces are calculated using a partitioned mobility matrix, comprising the transfer mobilities between noncollocated remote indicator DoF and the collocated driving point indicator DoF in a 3 fold over-determined 27x9 (rectangular) matrix (Eq. 5), denoted by the term 'combined' sensor array. All remote acceleration and sound pressure indicators are equally distributed across the surface of the receiver structure. A subset of remote sensors not included in the blocked force calculation is further used for subsequent component blocked force TPA and the assessment of experimental uncertainty. The latter may be related to assumptions imposed on the coupling interface dynamics, such as point-contact approximation or the neglect of rotational DoF.

Separated source (A): Separated from a rigid support structure, the free velocity of the vibration source (A) is measured according to ISO 9611 under idealized free boundary conditions. Premultiplication by the inverted driving point mobility matrix of the freely suspended source (see Figure 3(b)) yields the blocked force data (see Eq. (2)).

Target assembly (C'=A+B'): The independent blocked forces identified in the original assembly (C) and the separated source (A), respectively, are virtually coupled with the unconstraint suspended receiver structure (B') to construct a Virtual Acoustic Prototype. The corresponding physical target assembly ( $C^{\prime}$ ) enables validation of the synthesized prototype response (Eq. (8)) to assess uncertainty associated with transferability of the blocked force data.

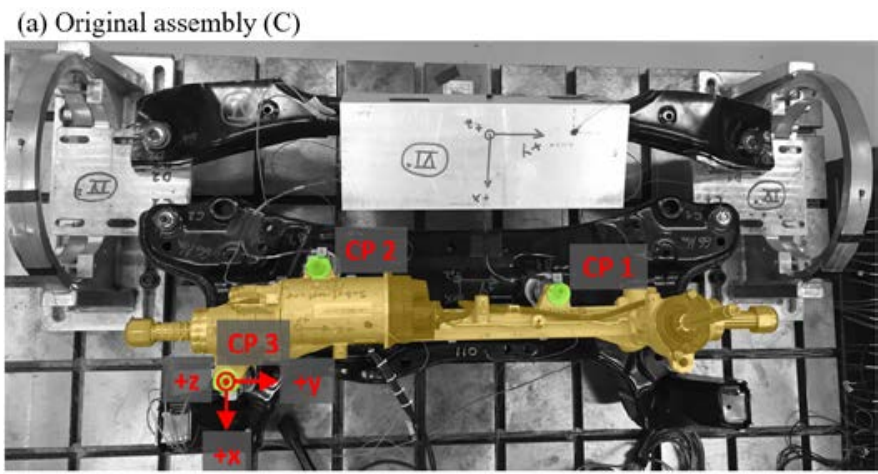

(b) Separated source (A)

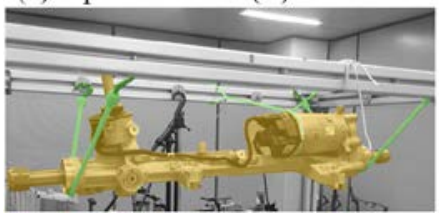

(c) Target assembly (C')

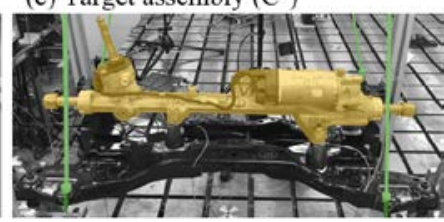

Figure 3. Measurement setups for the electric power steering system (source, orange): In-situ source characterization and component (blocked force) TPA in the original assembly (a); Free velocity characterization for the separated source (b); Virtual Acoustic Prototyping and Contribution Path Analysis in the target prototype assembly.

\section{Source characterization and accounting for uncertainty}

In theory, the invariant source characterization requires a complete interface description accounting for all translational and rotational DoF, as an incomplete description will most likely lead to an erroneous representation of the dynamics. In practical scenarios, restricted access to the coupling interface, limited measurement equipment or challenges associated with the determination of rotational DoF leads to neglecting particular coupling DoF. The importance of neglected coupling DoF and the degree to which an interface has been correctly described can quantitatively be assessed by the coherence style Interface Completeness Criteria (Eq. (9)) [10].The completeness description is evaluated between a set of

Page 5 of 12 
source-side artificial excitations and a single receiver-side DoF. For the investigated source structure the operational vibrations are assumed to originate from the electric motor. To achieve a realistic representation of the source mechanism the source-side artificial excitations are applied to the motor housing in different translational DoF. Any artificial force applied to the steering housing will fail to simulate the internal force mechanism which would cause a shifted load distribution at the coupling interfaces. In case of an incomplete interface description, an overstated external moment excitation would lead to a higher contributions of the rotational DoF, and the ICC would fail to represent the actual completeness for the operational state.

For the in-situ measurement in assembly (C) the ICC is evaluated for an arbitrary set of 9 source-side excitations to remote positions corresponding to the non-collocated sensor array. To consider the complexity of the internal force mechanism 10 different combinations of artificial source-side excitation applied at random positions are investigated. The overall ICC value, illustrated in Figure 4, indicates the completeness of the interface for the given excitation sets (row entry) to every single receiver-side DoF (column entry) expressed as single value. In theory, the criterion above yields 1 for a complete interface description. An overall value is approximated by summation of all values deviating from 1 and normalizing the result with respect to the number of frequency bins.

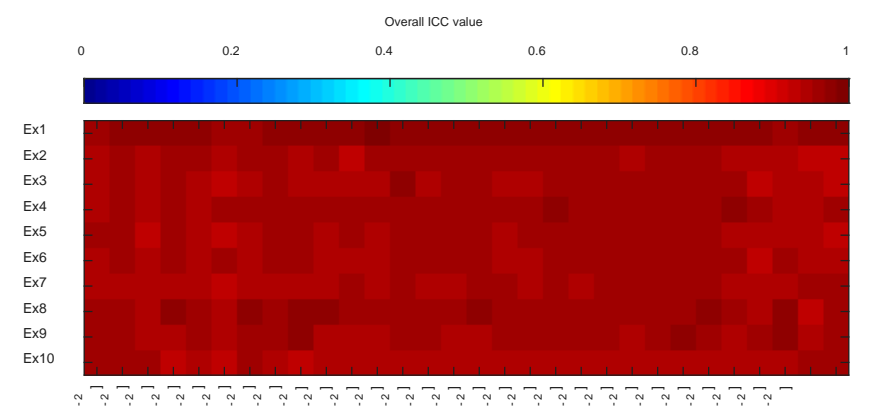

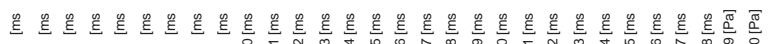

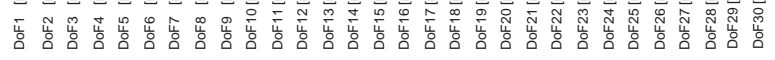

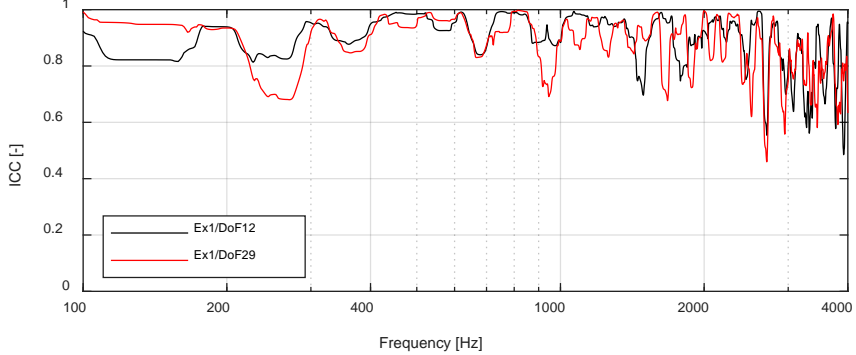

Figure 4. Matrix representation of the overall Interface Completeness Criterion (ICC) for 10 source-side excitation sets (row) to various indicator positions (column) in the original assembly (C) considering a frequency range between $100 \mathrm{~Hz}$ and $4 \mathrm{kHz}$ (top) and narrowband spectra of the ICC (bottom)

The matrix reveals the sensitivity of the criterion to the particular excitation and remote sensor location. The narrowband representation illustrates the ICC signature evaluated at a remote accelerometer and a microphone with a sufficient interface description in the range between $100 \mathrm{~Hz}$ and $2.5 \mathrm{kHz}$, contaminated to some extent by measurement uncertainty causing a perfect agreement to be unlikely. Beyond this range the completeness criteria decreases below 0.7 and indicates an incomplete interface description, likely due to the neglected rotational DoF and the breakdown of point-like connections. The translational blocked force has to be considered as Page 6 of 12 an erroneous representation of the source dynamics causing a VAP to be prone to error in this high frequency range.

The experimental completeness investigation is considered sufficient and confirms the validity of the simplified assumptions of point connected structures and neglected rotational DoF within the observed limited frequency range. The remaining uncertainty due to experimentation and data inconsistency is likely to be investigated in the on-board validation (Eq. (6)) [23]. The predicted acceleration responses in Figure 5 are in good agreement with the directly measured acceleration caused by a stationary artificial force excitation. A one-third octave band plot of the same data is included since this representation is mostly preferred for objective comparisons in industry. It was calculated by converting the narrowband results to one-third octave bands represented in Decibel. All predictions are based on the same transmitting paths so that relative errors above $2 \mathrm{kHz}$ are associated with the non-collocated blocked force calculation. The lack of severe artefacts suggests a consistency between the two separate stages of the in-situ blocked force characterization. Occasional deviations around some anti-resonances are observed and likely to be susceptible to user measurement error.

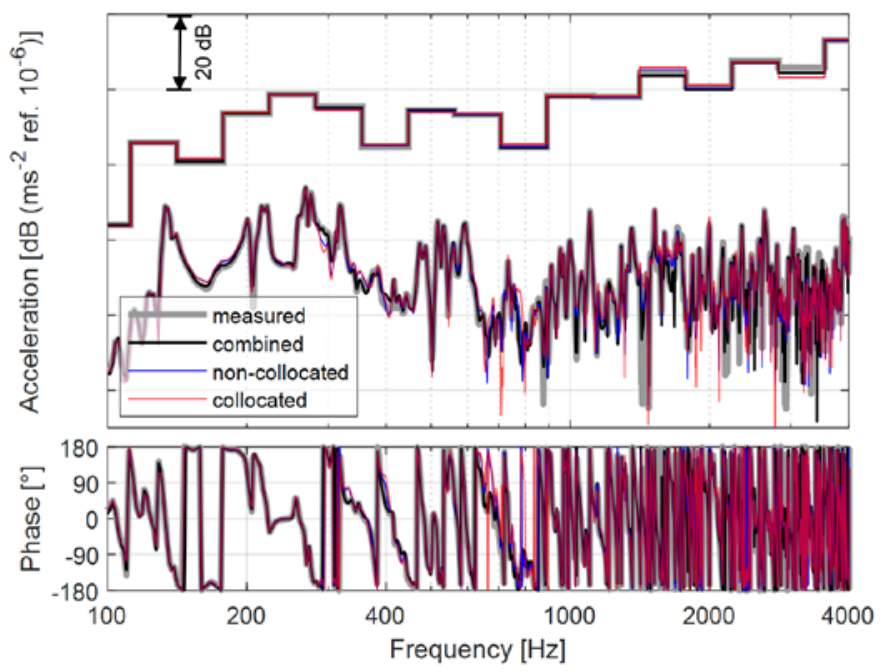

Figure 5. On-board validation results for original assembly (C) with artificial excitation at the motor housing: One-third octave and narrowband representation of amplitude (top) and phase spectra (bottom) for measured (grey), predicted/combined (black), predicted/non-collocated (blue) and predicted/collocated (red) structural response.

Although reasonable agreement is achieved for all predictive approaches, the 3 fold over-determined prediction based on a combined sensor array (black) indicates the least deviations to the measured signature, deviating no more than $3 \mathrm{~dB}$ beside the antiresonances. Therefore, the combined calculation is chosen as benchmark in further investigations. The agreement between collocated/determined and non-collocated/over-determined predictions suggests that the results are not effected by inversion error, usually suppressed through over-determination. However, an over-determined matrix can still suffer from large inversion error if not sufficiently conditioned. As frequency increases, deviations of the non-collocated calculation become more apparent in the narrowband representation, although following the general trend. In this region the determined prediction with collocated sensor positions appears to offer little improvement. Beyond $2 \mathrm{kHz}$ on-board validation (Eq. (6)) in general can be seen to under-predict the measured response. This suggests that the error is likely a result of neglected DoF, as this often manifests itself as an under-prediction. This result is in agreement with the previously investigated completeness criteria. 
To evaluate potential impact of inverse blocked force synthesis on the overall prediction accuracy, 8 additional remote positions were validated according to the component TPA framework (Eq. (7)). Having predicted multiple linear independent remote responses, assessment of the overall prediction accuracy for several different transmission paths is possible. Convenient comparison of all predicted TPA responses and the corresponding measured assembly responses is achieved by applying the Frequency Response Assurance Criteria (FRAC) and the Phase Assurance Criteria (PAC) [24] to derive a single-value correlation of any pair of measured and predicted remote response, representing the same input-output relationship. Identifying the systematic errors in the inversely determined blocked forces, 3x8 FRAC and PAC matrices are obtained, as illustrated in Figure 6.

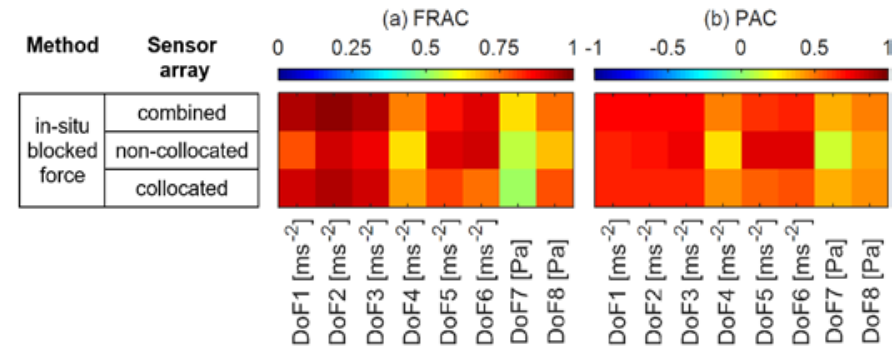

Figure 6. FRAC (a) and PAC (b) comparison of measured and predicted structural and acoustic responses in the frequency range between $100 \mathrm{~Hz}$ and $4 \mathrm{kHz}$ obtained in assembly (C) using component TPA.

Each row represents deviations from the predicted response to the directly measured acceleration or sound pressure for component TPA predictions with different sensor arrays. Perfect agreement, in theory, yields a value of 1 indicated by the red color code for identical magnitude and phase responses in the FRAC and PAC values, respectively. In contrast, a blue color code indicates magnitude mismatches or an inverted phase response.

From Figure 6, it can be found that satisfying approximation of the measured target response can be achieved within a multi-kHz frequency range with either of the calculation methods (Eq. (3-5)). The magnitude and phase prediction with combined and collocated sensor arrays are in good agreement, indicating superiority over the non-collocated setup. The relative poor correlation to the remote sound pressure measurements at DoF 7 and DoF 8 results from the shaker significantly contributing to these DoF due to extensive vibroacoustic coupling. Although, the hollow box was sealed with foam to minimize the effect of air-borne flanking during the characterization and validation process, it was observed that above $3 \mathrm{kHz}$ the measured pressure is dominated by flanking.

The previously observed high frequency deviation in the on-board validation (see Figure 5) or the systematically lower FRAC/ PAC values (see Figure 6) of the non-collocated calculation (Eq. (3)) are caused by contaminated blocked forces, illustrated in Figure 7(a). Beyond $2 \mathrm{kHz}$ the non-collocated blocked force results are clearly contaminated by an increasing amount of noisy deviations compared to the combined (Eq. (5)) and collocated variants (Eq. (4)). The indicator positions of the employed transfer mobilities are located further away from the active source component, resulting in a higher resemblance of the remote response and a loss of phase information [8]. This resemblance is indicated by a higher condition number of the non-collocated mobility matrix $\mathbf{Y}_{C, c b}^{T}$ for assembly (C), illustrated in Figure 8.

Page 7 of 12 (a) Combined / Non-collocated sensor array

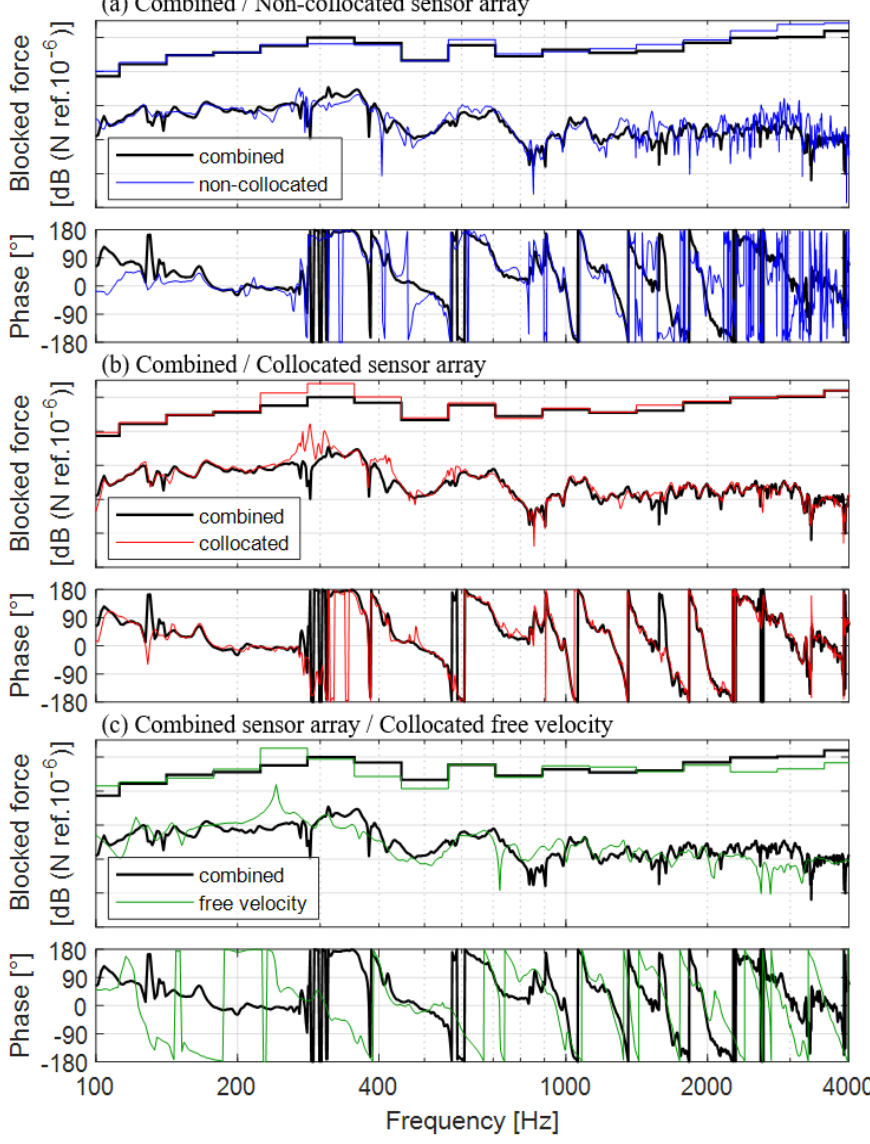

Figure 7. Comparison of narrowband and one-third octave blocked force spectra for in-plane direction x at CP3 predicted with different sensor arrays.

It is stressed that the large condition number at $241 \mathrm{~Hz}$ for the driving point matrix $Y_{A, c c}$ of the freely suspended steering gear coincides with an unwanted disturbance in the blocked force, as illustrated in Figure 7. Measurement noise or linear data dependence leads to illconditioning and the amplification of unwanted inversion errors.

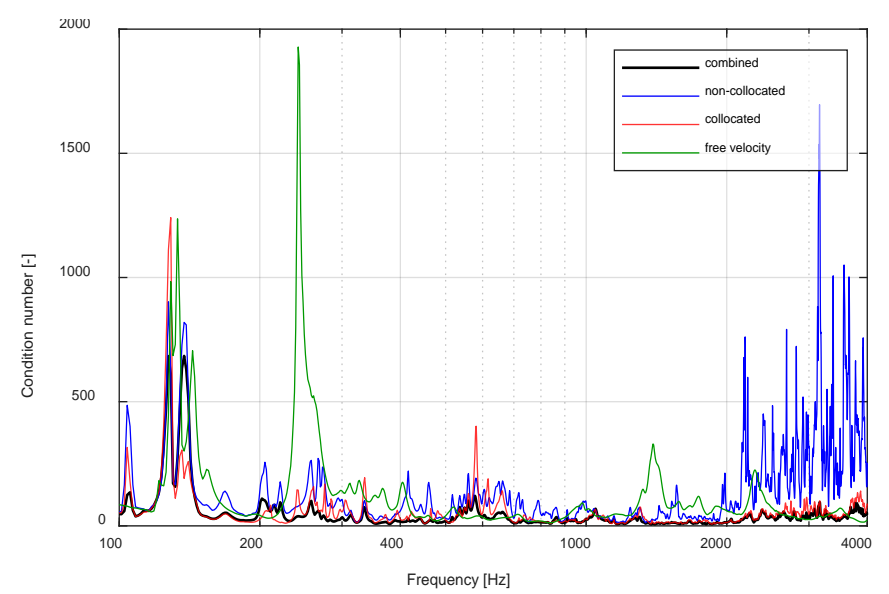

Figure 8. Sensitivity of the condition number to different degrees of overdetermination: 3 fold over-determined matrix (black); 2 fold non-collocated matrix (blue); determined collocated matrix (red) and determined source mobility matrix (green).

A comprehensive overview of magnitude and phase correlation for different blocked force DoF, using the combined calculation approach as reference, is illustrated in Figure 9. The good agreement 
between the combined and collocated blocked force characterization implies that the former mobility matrix is dominated by local system dynamics at the source interface described by the driving point measurements. Deviations of the blocked force obtained via free velocity method (Eq. (2)) arise due to the changed boundary conditions. Within the in-situ measurement the neglected rotational DoF are constrained by the receiver structure while the remaining translational DoF are blocked mathematically. This is particularly important as, although these physically constrained DoF may not contribute largely to the coupled response of the assembly, their blocking may significantly affect the determined blocked force in the remaining DoF. The significance of this alteration will be dependent upon the physical constraints on the assembly's coupling interface, which in case of the free velocity approach is completely unconstrained. In addition, the completeness criteria (Eq. (9)) cannot be applied to the uncoupled source structure assessing the contribution of the neglected rotational DoF in the free velocity characterization. Consequently, blocked forces obtained from the free velocity measurement differ slightly from the in-situ characterization over the entire frequency range.

(a) FRAC

\begin{tabular}{|c|c|}
\multicolumn{1}{|c}{ Method } & $\begin{array}{c}\text { Sensor } \\
\text { array }\end{array}$ \\
\begin{tabular}{|c|c|}
\hline in-situ & non-collocated \\
blocked & collocated \\
\hline free vel. & collocated \\
\hline
\end{tabular}
\end{tabular}

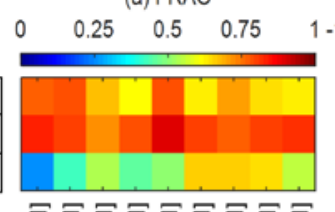

$\bar{z} z \bar{z} z \bar{z} z \underline{z}$

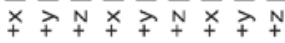

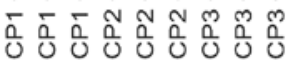

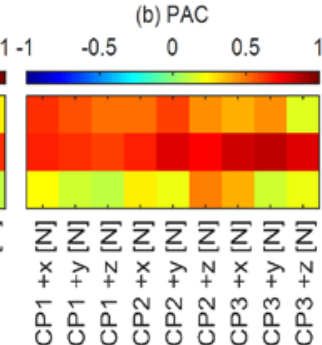

Figure 9. FRAC (a) and PAC (b) comparison of blocked forces identified as per ISO/DIS 20270 and ISO 9611, using data from different sensor arrays in the frequency range between $100 \mathrm{~Hz}$ and $4 \mathrm{kHz}$ obtained in assembly (C).

\section{Virtual Acoustic Prototyping and auralization}

Satisfying on-board validation results and a complete interface description are indicators for the transferability of the obtained blocked forces. Since the setup does not account for all DoF, the blocked forces should only be transferred between assemblies of similar coupling dynamics, i.e. rigid to rigid or resilient to resilient, to ensure the representation of the source characteristics in some other scenario [7]. This is particularly important with the construction of the VAP in mind, as one would be aiming to reproduce the assembly response in the most realistic way possible.

To ensure dynamically similar coupling conditions with respect to the original assembly (C), particularly at the sensitive source-receiver interface, a freely suspended subframe (B') is used as the intended target installation (C'). Similarity of the mounting conditions between in-situ characterization and VAP construction can experimentally be assessed by the Interface Completeness Criteria (see Figure 10), while similar considerations are not possible for the transfer of free velocity data (Eq. (2)). According to the previous completeness investigation an equivalent set of 10 source-side artificial excitations is applied to the electric motor while the response is evaluated at 7 remote points on the receiver subframe. Both the matrix of the overall ICC value and the narrowband representation indicate a similar completeness characterization akin to the original assembly (C) revealing an insufficient interface description above $2.5 \mathrm{kHz}$. Although rotational DoF are neglected, the ability to reconstruct the remote response is retained.

Page 8 of 12
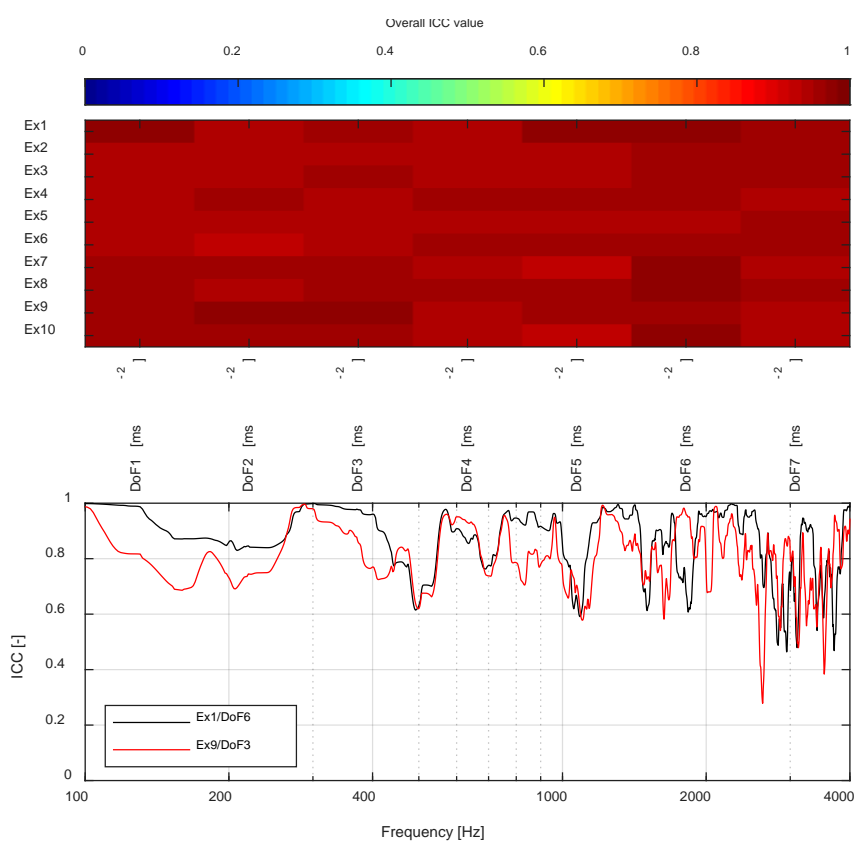

Figure 10. Matrix representation of the overall Interface Completeness Criterion (ICC) for 10 source-side excitation sets (row) to various indicator positions (column) in the target installation (C') considering a frequency range between $100 \mathrm{~Hz}$ and $4 \mathrm{kHz}$ (top) and narrowband spectra of the ICC (bottom)

All remaining uncertainty in the blocked forces will, however, be propagated through the virtually coupled receiver structure and become apparent in the physical validation of the VAP. The predicted acceleration in the virtual assembly (see Figure 11) is in reasonable agreement with the measured response of the physical prototype C' confirming the transferability of the independent blocked forces.

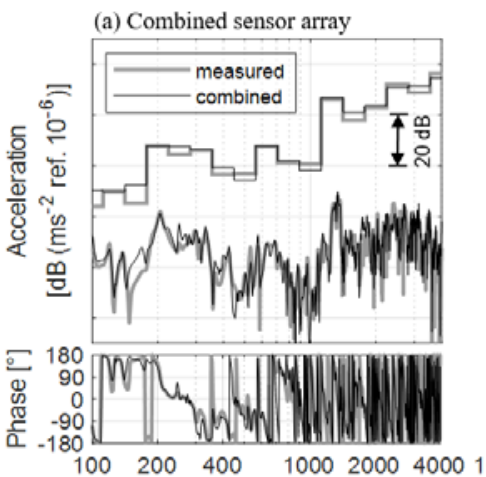

(b) Non-collocated sensor array
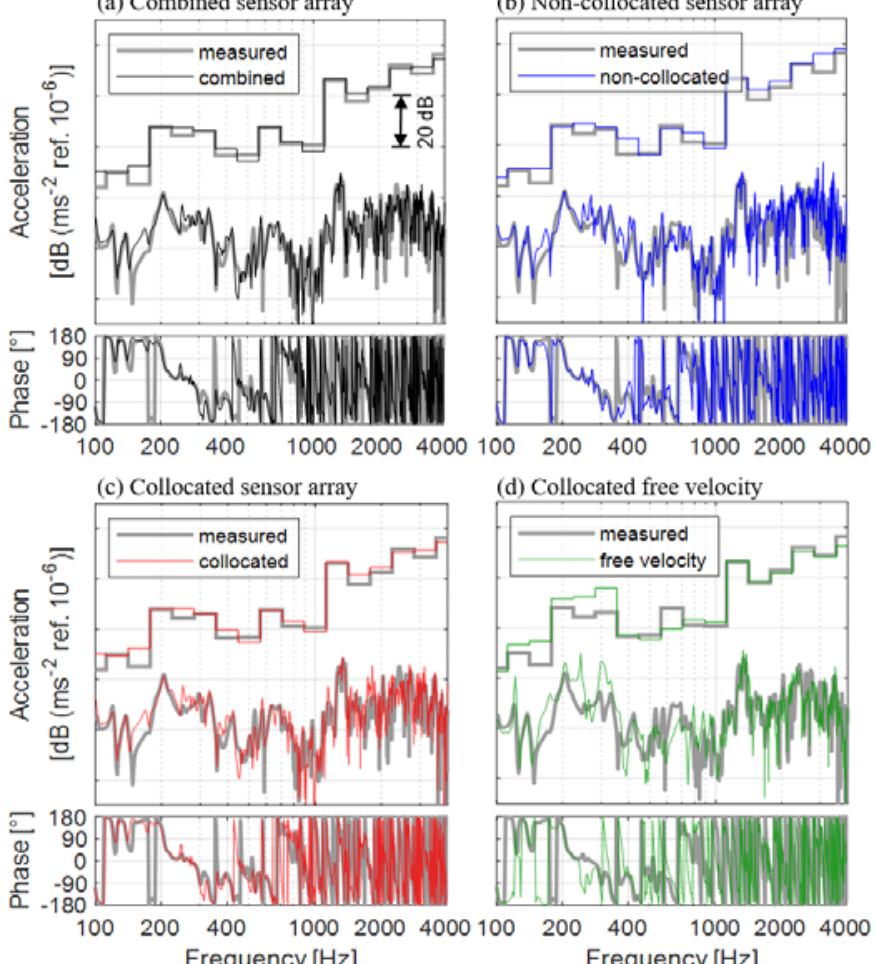

Frequency $[\mathrm{Hz}$

Frequency $[\mathrm{Hz}]$

Figure 11. One-third octave and narrowband representation of VAP-predicted vibration responses in the virtual assembly (C') compared to measured responses from artificial excitation on the motor housing. 
The transferability is restricted to the frequency limit of $2.5 \mathrm{kHz}$ due to the completeness of the interface, indicated by gradually increasing deviations in the predicted acceleration (see Figure 11). In addition, the prediction and corresponding validation in the virtual prototype pertain the problem of identifying potential errors in the free velocity setup, since redundant blocked force data are usually not available for pair-by-pair comparisons. The highlighted large condition number of the free source mobility $Y_{A, c c}$ (see Figure 8) and the associated inversion error affects the predicted acceleration to exceed the measured reference.

Besides objective evaluation the subjective impression is a fundamental requirement for robust $\mathrm{NVH}$ engineering. Appropriate post-processing of the blocked force spectra allows for time domain reconstruction and subsequent auralization. Such vibration and sound pressure time signatures are well suited for any type of data analysis including subjective evaluation of the product sound quality. For auralization purposes the steering gear is operated in alternating directions with linearly increased rack speed to generate a timevariant system excitation. The actuation of the steering gear is directly controlled via the three phases of the permanent magnet synchronous motor to deliberately accentuate the harmonics of the electric motor and to avoid the optimized control of the electronic control unit [22].

Within the VAP auralization blocked forces are obtained by combining the time-variant system responses with the previously described mobility matrices. Sequential Fourier-spectra retain the time structure of the blocked force, thus providing a time domain source characterization using the Overlap-add method [25]. To account for the limited frequency range of the FRFs a bandpass filter for zero-phase digital filtering is applied to all predicted and measured time domain signals. In Figure 12 the blocked force time signatures are used to synthesize remote accelerations in the virtual environment (C').
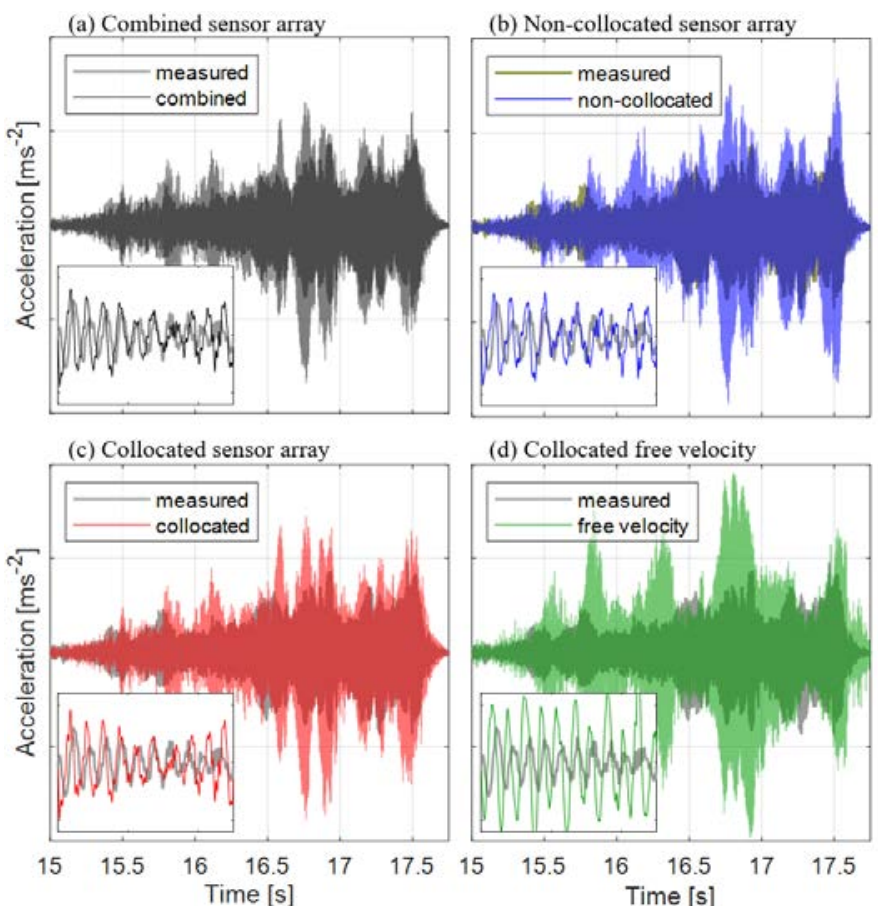

(d) Collocated free velocity

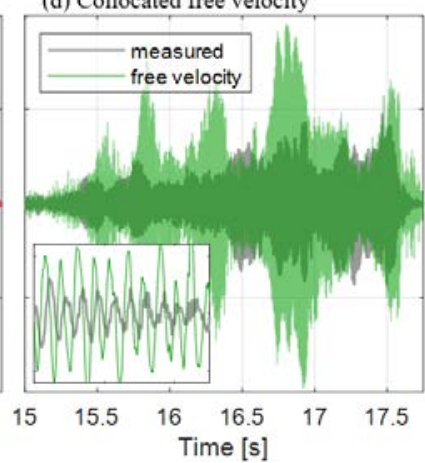

Figure 12. Measured and synthesized time domain assembly responses in the actual prototype (C') for a simulated ramp-up obtained from operational measurements with different sensor arrays. The inset spans the interval between $15.5 \mathrm{~s}$ and $15.55 \mathrm{~s}$.

Page 9 of 12
A similar agreement of the time signature and the measured prototype response is illustrated in Figure 12, akin to the VAP prediction for the stationary artificial excitation in Figure 11. With exception of the free velocity method, the temporal structure is predicted with good accuracy, leading a realistic auralization. It is noted that the timevarying VAP validation assumes repeatable operational conditions for the in-situ and free velocity approach to ensure consistent system responses. However, slight deviations in the source mechanism are propagated through the blocked forces or affect directly the reference measurement of the prototype. These source variations are superimposed with the previously mentioned uncertainties.

The time domain results for the collocated sensor array (Eq. (4)) and the free velocity (Eq. (2)) characterization are replicated in Figure 13 in the form of Campbell diagrams. Both predictive approaches bare the same number of driving point measurements and thus the same experimental effort to identify the mobility matrix of either the coupled assembly $Y_{C, c c}$ or the isolated source structure $Y_{A, c c}$. The slight over-prediction (see Figure 13)) of the free velocity approach, notably those of Figure 11(d), is also apparent in the harmonics of the electric motor in the frequency range below $400 \mathrm{~Hz}$. With regards to the construction of a VAP the collocated in-situ characterization presents the more accurate reconstruction of the measured harmonics.

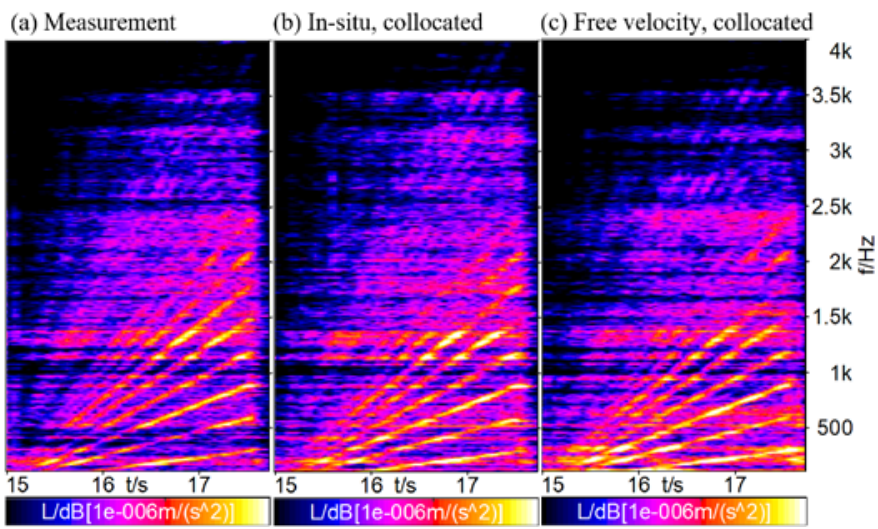

Figure 13. Campbell diagrams of assembly (C') acceleration responses: measured (a); synthesized using in-situ blocked force method (ISO/DIS 20270) with collocated sensor array (b); synthesized from free velocity (ISO 9611)

Developing superior NVH quality requires not only engineering techniques for response prediction in virtual assemblies but also tools to perform diagnosis and identification of structure-borne sound in fully assembled structures. Together, the above can provide valuable insight into the propagation of forces for design optimization. The obtained measurements in the prototype assembly (C'), (i.e. operational velocity and assembly FRF matrix) can further be used for advanced diagnosis employing Component Transfer Path Analysis, for example to conduct contribution analysis to study the transmission of structure-borne sound in assembled structures.

For analysis of the partial path contributions, typically the quantified operational blocked loads are combined with particular propagating transfer paths, so as to predict the assembly response on receiver-side target DoF. This procedure allows assigning complex partial path contributions from the individual contact interface DoF to the overall structure borne sound and vibration response at defined target points. It is this detailed information which makes the method useful for troubleshooting $\mathrm{NVH}$ problems. In practice, the predicted partial path contributions are considered to be trustworthy if target accelerations measured during operation of the REPS (source) are in good 
agreement with the predicted total contribution, the latter calculated as complex sum over all individual partial paths (see Figure 14). In the example provided in Figure 14, best contribution analysis results are achieved with the in-situ blocked force method for the combined sensor array. The directly measured reference response at a selected target point on the subframe-side of assembly (C') coincides with the (virtually) predicted sum acceleration as illustrated in the first and second row of Figure 14(a). In a practical NVH engineering context, this partial contribution may be of interest with respect to evaluating potentials to improve or diagnose $\mathrm{NVH}$ performance criteria, e.g. by identifying critical frequency ranges with exceeding contributions to target DoF.

The bar graph in Figure 14(b) illustrates the total averaged acceleration level in Decibel (predicted (black), measured (red)) with a total prediction error of less than $1 \mathrm{~dB}$. The diagram also provides a rank ordering of all partial transfer paths, facilitating identification of dominant paths. In the given example, all paths contribute comparable to the target response, with the contribution from the in-plane DoF at coupling point $1\left(\mathrm{CP} 1 \_+y\right)$ being the least significant one.

(a) Partial path contribution

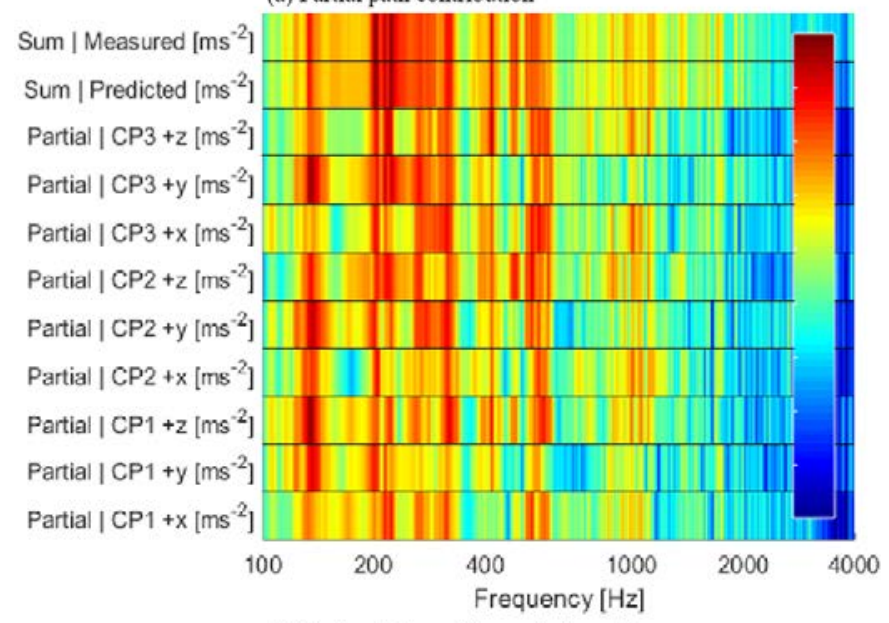

(b) Rank ordering of transmission path

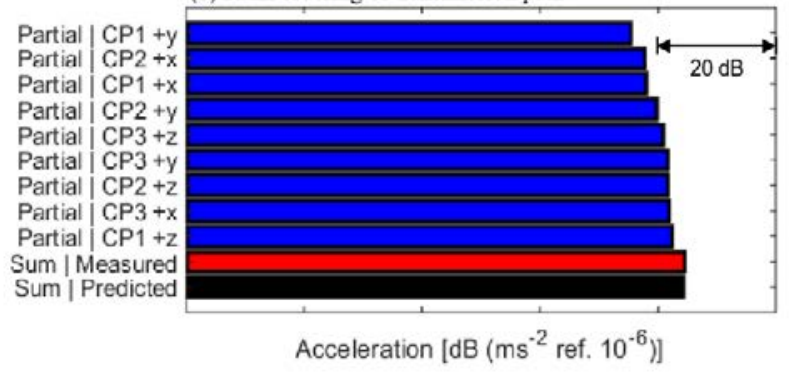

Figure 14. Narrowband results of contribution analysis for total predicted sum and partial path contributions from the coupling DoF to a target response DoF (a) and rank ordered partial contributions (b) for the operated REPS (ramp-up) in target assembly (C').

Concerning the practical interpretation of the partial contribution it is mentioned that the illustrated plot offers no insight into whether a significant contribution to the target is caused by a sensitive transmission path or a high dynamic loading at the coupling DoF. For example, a high FRF would be evident for a sensitive transmission path of the coupled system not clearly indicating whether modifications should be applied to the source or receiver-side of the structure.

Page 10 of 12
Contribution analysis was also performed with inversely determined blocked forces from data obtained with collocated and non-collocated sensor arrays using the in-situ blocked force approach (ISO/DIS 20270; Eq. (4) / Eq. (3)) and the free velocity approach (ISO 9611; Eq. (2)), respectively. A comparison of the total predicted contribution sums is provided in Figure 15.

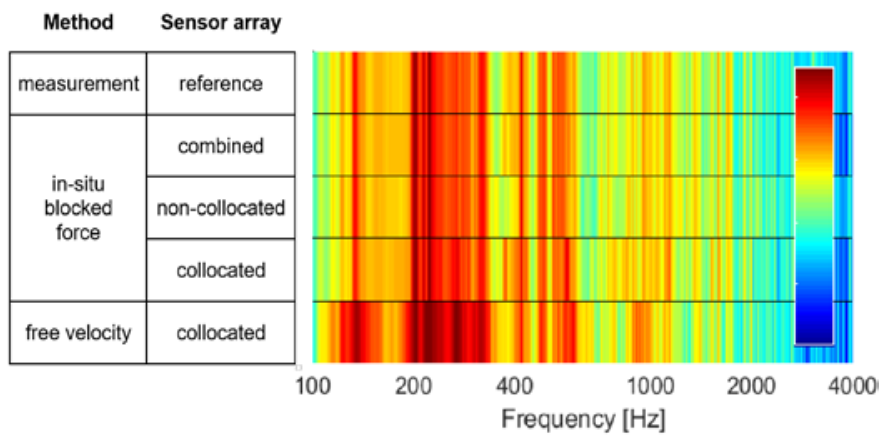

Figure 15. Comparison of measured narrowband acceleration at the indicator point and the total predicted remote response for the inverse force synthesis of the ISO/DIS 20270 and the ISO 9611 for the simulated ramp up of the REPS.

Satisfying prediction results can be achieved with all in-situ blocked force source characterization approaches (ISO/DIS 20270), with data from the collocated sensor array (Eq. (4)) yielding almost identical prediction results as the combined sensor array (Eq. (5)). However, the prediction accuracy achieved with the free velocity approach (Eq. (2)), is significantly worse than in any other in-situ blocked force approach. This is due to the transferred translational blocked forces fail to represent the dynamic properties of the vibration source operated in assembly (C') due to the neglected of rotational DoF and the missing physical constraints during the source characterization with the source separated from a receiver.

\section{Summary/Conclusions}

This paper has been concerned with the exploration of potentials to reduce complexity in agreed experimental methods for the characterization of structure-borne sound and vibration sources, with the objective to increase industrial practicability of component (blocked-force) Transfer Path Analysis (TPA) and Virtual Acoustic Prototyping (VAP) in the automotive sector.

A framework has been presented facilitating prediction, diagnosis and auralization of sound and vibration in assembled structures employing independent source data from free velocity (ISO 9611) and in-situ blocked force (ISO/DIS 20270) measurements of different complexity. Practical procedures, such as on-board validation (OBV) and Interface Completeness Criteria (ICC), have been adopted in the framework to address uncertainties related to consistency, completeness and transferability of experimental source data; the latter required to conduct component (blocked-force) Transfer Path Analysis (TPA) and Virtual Acoustic Prototyping (VAP).

A Rack-and-pinion Electric Power Steering (REPS) system inducing structure-borne sound and vibration into a rigidly connected subframe receiver, has been used to experimentally validate the procedures of the framework. The sensitivity of inversely determined blocked force source data, from measurements in-situ (ISO/DIS 20270), on sensor placement and sensor number has been investigated and benchmarked against blocked force source data from free velocity measurements (ISO 9611) in terms of industrial feasibility. 
For the investigated case, it has been found that, when using collocated sensor arrays, i.e. indicator and contact degrees of freedom coincide, the experimental effort of the in-situ blocked force method can be reduced to the benchmark, while prediction accuracy is superior. With reference to the best achieved source characterization result, by solving the 3-fold over-determined (in-situ blocked force) inverse problem, significant experimental simplification has been attained without rigorous impairing accuracy.

Uncertainties imposed by assumptions on coupling interface discretization (point-like contact interface, translational coupling DoF) have been experimentally addressed by applying the Interface Completeness Criteria (ICC). Applying ICC to assess completeness in coupled structures has been found to be relatively simple since only additional excitation on the source-side of the assembly is required which in essence is suggested as part of a preliminary validation test with artificial excitation by ISO/DIS 20270. Assessment of the coherence-style ICC has confirmed validity of the experimental approximated interface conditions within a sufficient frequency range. Applicable only for coupled structures, it has not been possible to assess completeness of source data obtained from free velocity measurement (ISO 9611) under unconstrained boundary conditions.

All obtained blocked force source data has been utilized for component (blocked force) TPA and VAP to predict, auralize and diagnose REPS-induced structure-borne sound and vibration in the actual and a virtual subframe installation, respectively. Uncertainty in the VAP predictions related to transferability of source data has been assessed by comparing predicted receiver responses with actual measurements. It has been found that employing operational blocked forces from in-situ characterization (ISO DIS/20270) provides realistic representation of the REPS system virtually operated on the target subframe within a multi-kHz frequency range. Representativeness of the receiver in the initial source characterization assembly to the target receiver-installation has been found significant to ensure transferability of the independent blocked force due to similar local coupling dynamics. It has been concluded that VAP prediction employing blocked forces from free velocity measurements (ISO 9611) suffers from transferability issues resulting from altered constraints on the coupling interface during source characterization and target prediction step, yielding less accurate prediction results.

Contribution analysis in virtual vehicle environments together with VAP auralization of corresponding assembly responses has been demonstrated to be valuable diagnostic and system engineering tools, allowing assessment of objective and subjective NVH performance in virtual prototypes. It is advisable to conduct source characterization with the in-situ blocked force approach, specified in ISO/DIS 20270, since receiver-representativeness can be achieved by designing special receivers according to the target installation, thus reducing uncertainty related to transferability of blocked force source data. In addition, the possibility to apply on-board validation in combination with the Interface Completeness Criteria, contributes towards increasing robustness of component (blocked force) TPA and Virtual Acoustic Prototyping in industrial applications.

\section{References}

1. Moorhouse, A.T., "Virtual Acoustic Prototypes: Listening to machines that don't exists,” Acoustics Australia 33(3):97, 2005.
2. Sturm, M., Alber, T., Moorhouse, A.T., Zabel, D. et al., “The insitu blocked force method for characterization of complex automotive structure-borne sound sources and its use for virtual acoustic prototyping," Presented at ISMA 2016, Belgium, September 19-21, 2016.

3. van der Seijs, M.V., de Klerk D., and Rixen D.J., "General framework for transfer path analysis: History, theory and classification of techniques,” Mechanical Systems and Signal Processing 68 (217-244), 2016.

4. de Klerk, D., Rixen, D.J., and Voormeeren, S.N., “General framework for dynamic substructuring: History, review and classification of techniques,” AIAA Journal 46 (5):1169-1181, 2008.

5. The International Organization for Standardization (ISO), "Acoustics - Characterization of sources of structure-borne sound with respect to sound radiation from connected structures - Measurement of velocity at the contact points of machinery when resiliently mounted,” BS ISO 9611:1996, 1996.

6. Moorhouse, A.T., Elliott, A.S., and Evans, T.A., "In situ measurement of the blocked force of structure-borne sound sources,” Journal of Sound and Vibration 325 (4-5):679-685, 2009.

7. The International Organization for Standardization (ISO), "Acoustics - Characterization of sources of structure-borne sound and vibration - Indirect measurement of blocked forces,” ISO/DIS 20270:2018(E), 2018.

8. Wernsen, M.W.F., van der Seijs, M.V., and de Klerk, D., “An indicator sensor criterion for in-situ characterisation of source vibrations,” In: Sensors and Instrumentation, Volume 5, pp. 5569. Springer, Cham, 2017.

9. Meggitt, J.W.R., Elliott, A.S., and Moorhouse, A.T., “A covariance based framework for the propagation of uncertainty through inverse problems with an application to force identification,” Mechanical Systems and Signal Processing, 2018.

10. Meggitt, J.W.R., Moorhouse, A.T., and Elliott, A.S., "On the problem of describing the coupling interface between substructures: An experimental test for 'completeness',' In: Dynamics of Coupled Structures, Volume 4, pp. 171-182. Springer, Cham, 2018.

11. Elliott, A.S., Moorhouse, A.T., and Pavić, G., "Moment excitation and the measurement of moment mobilities," Journal of Sound and Vibration 331(11):2499-2519, 2012.

12. Moorhouse, A.T., Evans, T.A., and Elliott, A.S., "Some relationships for coupled structures and their application to measurement of structural dynamic properties in situ," Mechanical Systems and Signal Processing 25(5):1574-1584, 2011.

13. van der Seijs, M., van den Bosch, D., Rixen, D., and de Klerk, D., "An improved methodology for the virtual point transformation of measured frequency response," COMPDYN, 2013.

14. de Klerk, D., and Rixen, D.J., "Component transfer path analysis method with compensation for test bench dynamics," Mechanical Systems and Signal Processing 24(6):1693-1710, 2010.

15. Elliott, A.S., Moorhouse, A.T., Huntley, T., and Tate, S., "Insitu source path contribution analysis of structure borne road noise," Journal of Sound and Vibration 332(24):6276-6295, 2013.

16. Lennström, D., Olsson, M., Wullens, F., and Nykänen, A., "Validation of the blocked force method for various boundary conditions for automotive source characterization,” Applied Acoustics 102:108-119, 2016. 
17. Alber, T., Sturm, M., and Moorhouse, A.T., "Independent characterization of structure-borne sound sources using the insitu blocked force method," Presented at Internoise 2016, Germany, August 21-24, 2016.

18. Sturm, M., Moorhouse, A.T., Yankonis, M., Marchand ,C. et al., "Robust NVH development of steering systems using in-situ blocked forces from measurements with low-noise driver simulators,” Presented at NOISE-CON 2017, USA, June 12-14, 2017.

19. Moorhouse, A.T., "Virtual acoustic prototypes as a tool for lownoise design," Proceedings of the Institute of Acoustics 24(4), 2002.

20. Meggitt, J.W.R., "On in-situ methodologies for the characterisation and simulation of vibro-acoustic assemblies,” Ph.D., University of Salford, Salford, United Kingdom, 2017.

21. van der Seijs, M., Pasma, E.A., de Klerk, D., and Rixen D.J., “A robust transfer path analysis method for steering gear vibrations on a test bench,” Presented at ISMA 2016, Belgium, September 7-9, 2014.

22. Kimpián, T. and Augusztinovicz, F., "Multiphase multisine signals - Theory and practice," Presented at ISMA 2016, Belgium, September 19-21, 2016.

23. Moorhouse, A.T. and Meggitt, J.W.R., "Evaluation of uncertainties in classical and component (blocked force) transfer path analysis (TPA),” SAE Technical Paper, 2019.

24. Knechten, T., Morariu, M.-C., and van der Linden, P.J.G., "Improved method for frf acquisition for vehicle body NVH analysis,” SAE Technical Paper 2015-01-2262, 2015.

25. Meggitt, J.W.R., Elliott, A.S., Moorhouse, A.T., Banwell, G. et al., "Broadband characterisation of in-duct acoustic sources using an equivalent source approach,” Journal of Sound and Vibration (442):800-816, 2019.

\section{Contact Information}

Michael Sturm, Ph.D., AS/ENV-NA, Robert Bosch Automotive Steering LLC, 15000 Haggerty Road, Plymouth, MI 48170, USA. e-mail: michael.sturm2@bosch.com

\section{Definitions/Abbreviations}

$\begin{array}{ll}\text { DoF } & \text { Degrees of freedom } \\ \text { FRF } & \text { Frequency response function } \\ \text { ICC } & \begin{array}{l}\text { Interface Completeness } \\ \text { Criteria }\end{array} \\ \text { NVH } & \text { Noise, Vibration and } \\ \text { OBV } & \text { Harshness } \\ \text { REPS } & \text { On-board validation } \\ & \text { Rack-and-pinion Electric } \\ \text { TPA } & \text { Power Steering system } \\ \text { VAP } & \text { Transfer Path Analysis } \\ \end{array}$

